Advances in Radio Science (2003) 1: 325-327

(C) Copernicus $\mathrm{GmbH} 2003$

\title{
The background absorption at high latitudes
}

\author{
M. Harrich ${ }^{1}$, M. Friedrich ${ }^{2}$, S.R. Marple ${ }^{3}$, and K.M. Torkar ${ }^{1}$ \\ ${ }^{1}$ Space Research Institute, Austrian Academy of Sciences, Schmiedlstrasse 6, A-8042 Graz, Austria \\ ${ }^{2}$ Dept. of Communications and Wave Propagation, Technical University Graz, Inffeldgasse 12, A-8010 Graz, Austria \\ ${ }^{3}$ Dept. of Communication Systems, Lancaster University, Lancaster, LA1 4YR, UK
}

\begin{abstract}
The high latitude ionosphere is more often disturbed than quiet, i.e. fluxes of charged particles dominate over solar controlled ionisation. Nonetheless, the actual amount of the predictable, solar-controlled ionisation and the resulting absorption is of interest for some applications.
\end{abstract}

\section{Introduction}

Absorption of natural extraterrestrial radio sources is routinely measured by riometers at many locations in polar regions. According to magneto-ionic theories of different degrees of complexity (Appleton-Hartree, Sen and Wyller, 1960, or the Extended Sen and Wyller, Friedrich et al., 1991) to a good approximation absorption is proportional to the product of electron density $N_{e}$ and the frequency of collisions between electrons and neutrals $v_{n e}$. According to laboratory measurements (Phelps and Pack, 1959) $v_{n e}$ is proportional to pressure. The product of $N_{e} v_{n e}$ thus maximises in the $D$ region. Since pressure deviates only marginally relative to predictable "normal" values, the $D$-region electron densities must be responsible for variations of radio wave absorption.

\section{Riometer}

A riometer measures signal strength of natural extraterrestrial radio sources at frequencies which always penetrate the ionosphere $(>20 \mathrm{MHz})$. An upward looking antenna can "see" different parts of the sky in the course of a sidereal day (Quiet Day Curve). Negative deviations from the QDC are attributed to absorption by the ionosphere. Riometers are typically located in polar regions, i.e. beyond the arctic circle and therefore in Winter in full darkness for the whole sidereal day. In mid-Summer, however, the solar zenith angle $\chi$ can vary between $46^{\circ}$ and $88^{\circ}$. The imaging riometer IRIS located in Kilpisjrvi, Finland, has 49 beams, whereof the central (upward looking) beam is the narrowest $\left( \pm 6.3^{\circ}\right)$. The

Correspondence to: M. Friedrich

(friedrich@inw.tu-graz.ac.at) installation operating on $38.2 \mathrm{MHz}$ is very carefully maintained, repeatedly calibrated and the QDC established every two weeks. Due to the absorption of the solar controlled ionosphere, the course of the received signal on a quiet Summer day should ideally be systematically smaller than that of an equally quiet Winter day. To put his idea to a test we plot such QDC's vs. sidereal time and form the difference between the two (Fig. 1). This difference (mean of AM and PM) indeed varies with solar zenith angle (see results in Fig. 5), however repeating the procedure for high solar activity yields an inverse behaviour, namely that there is less absorption for larger solar activity. We therefore conclude that this method of establishing the background absorption of the order of typically less than $0.2 \mathrm{~dB}$ requires a stability exceeding what is achievable with even the most carefully maintained instrument. Furthermore this procedure could at best yield absorption beyond the very small night values, but can not yield night time absorption itself.

\section{Rocket data}

The least questionable method to establish absolute electron densities is to employ a semi-in situ wave propagation method, i.e. to transmit RF waves from the ground to the flying rocket. Of the 125 such profiles measured by rocket flights in the auroral zone, only about a quarter cover a height region which one can reasonably expect to contribute in excess of $90 \%$ to the radiowave absorption; from experience of looking at the simulated incremental absorption $\left(\mathrm{dB} \mathrm{km}^{-1}\right)$ we set the minimal height coverage limit to 70 to $110 \mathrm{~km}$. By definition a riometer measures excess absorption, i.e. signals below the experimentally determined QDC; hence subtracting the (measured) riometer absorption from the simulated integral absorption should yield the absorption due to the undisturbed ionosphere (solar controlled "rest absorption"). Figure 2 shows these absorption differences as a function of solar zenith angle. As one can see, in many instances the (riometer) absorption measured on the ground was larger than the simulated (integral) absorption (i.e. negative rest 


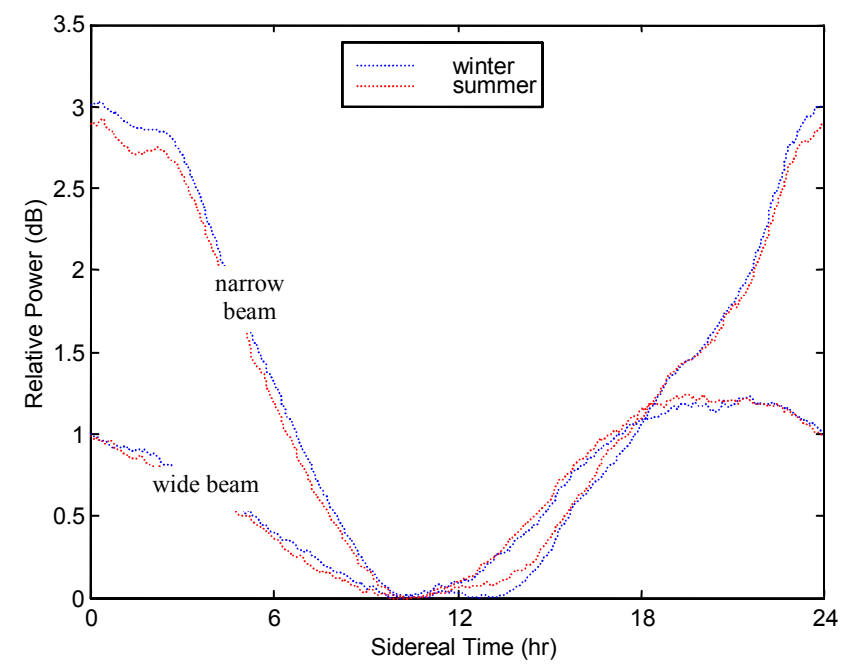

Fig. 1. Empirically determined quiet day curves of the IRIS riometer in Winter and Summer.

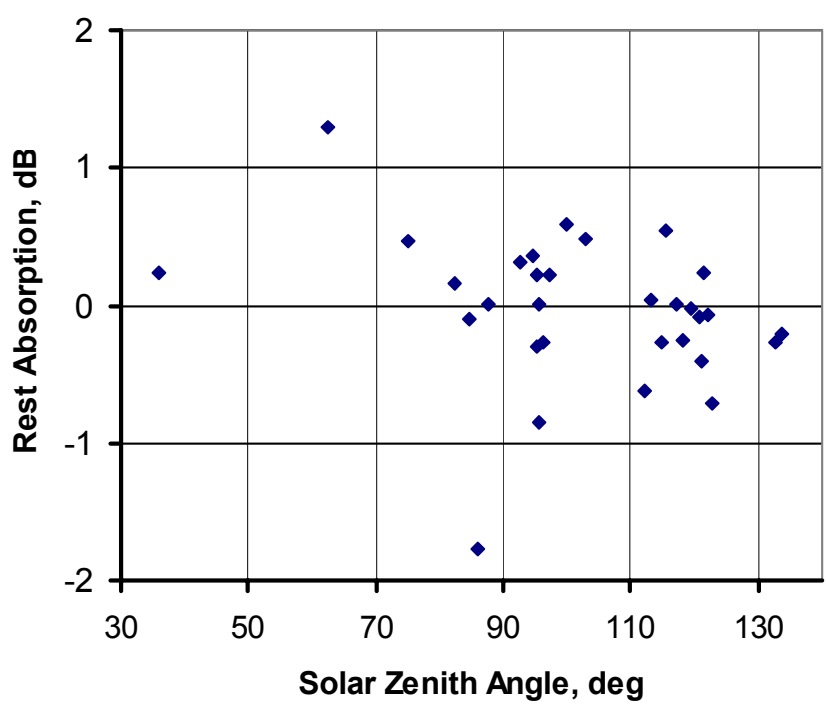

Fig. 2. Difference between simulated integral absorption and observed riometer absorption as a function of solar zenith angle (calculated for $27.6 \mathrm{MHz}$ and $\mathrm{x}$-mode).

absorption!). Apart from trivial explanations such as inaccurately determined QDC's, poor riometer readings or insufficient height coverage of the electron density profiles, one can conclude that the electron density measured by the sounding rocket is not always representative for the part of the ionosphere "seen" by the riometer.

\section{True Quiet ionosphere}

Apparently none of the above methods is suited in practice to determine the rest absorption, therefore another approach is pursued. The European incoherent scatter radar facility EISCAT located near Troms $\emptyset$ has provided literally thousands of electron density profiles. The height resolution is poorer

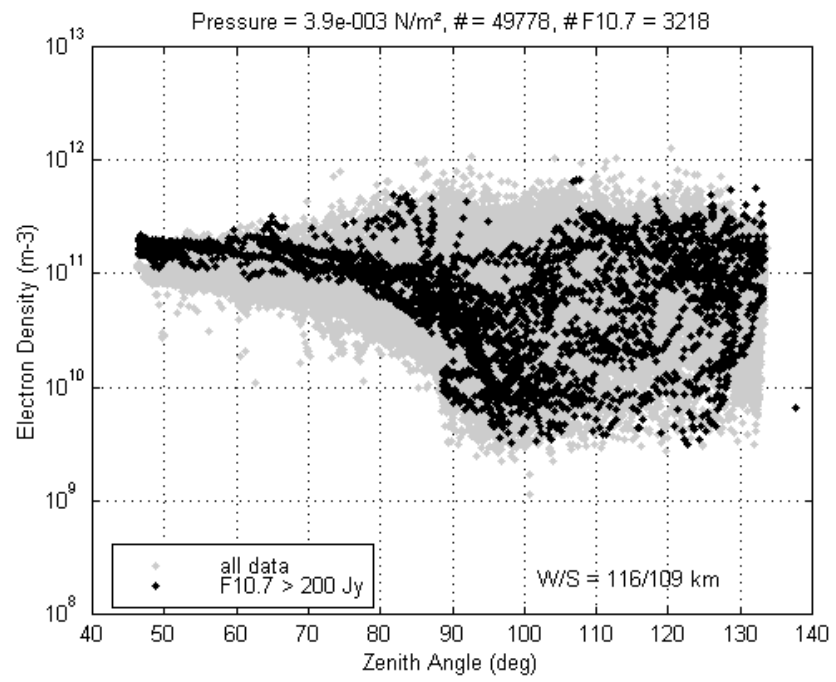

Fig. 3. E-region electron densities as a function of solar zenith angle. Note that probably at night values below the EISCAT threshold exist. Values for high solar activity are highlighted.

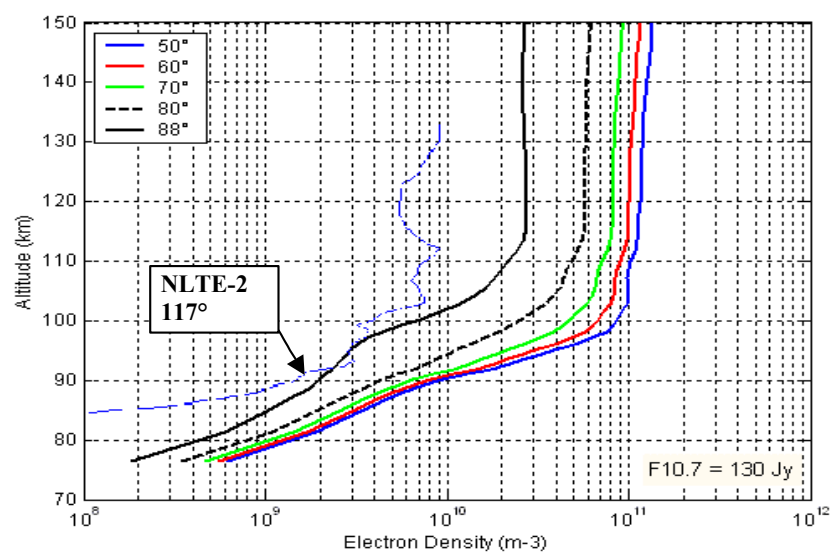

Fig. 4. True Quiet electron densities for medium solar activity and various solar zenith angles. The profile of the rocket flight NLTE-2 is also included as a proxy for night-time True Quiet.

than that of most rocket borne methods and also the threshold density between typically at $3.10^{8} \mathrm{~m}^{-3}$ and $2.10^{9} \mathrm{~m}^{-3}$ for VHF and UHF, respectively, is considerably larger than what is achievable by sounding rockets measurements. We now plot all available high latitude electron density data at many pressures surfaces as a function of solar zenith angle; Fig. 3 shows an example of the $E$-region. The intention behind plotting the values at pressure surfaces rather at constant heights is that by this procedure seasonal variations are at least in part accounted for; for the present analysis we use CIRA-86 corrected by local atmospheric measurements made above Andøya. The figure shows (a) a large variability notably at night and (b) a distinct boundary during the day. This boundary density seems to follow a relation with solar zenith angle as described by the Chapman behaviour of the form $N_{e}=N_{o}(\cos \chi)^{n}$. We repeat this 


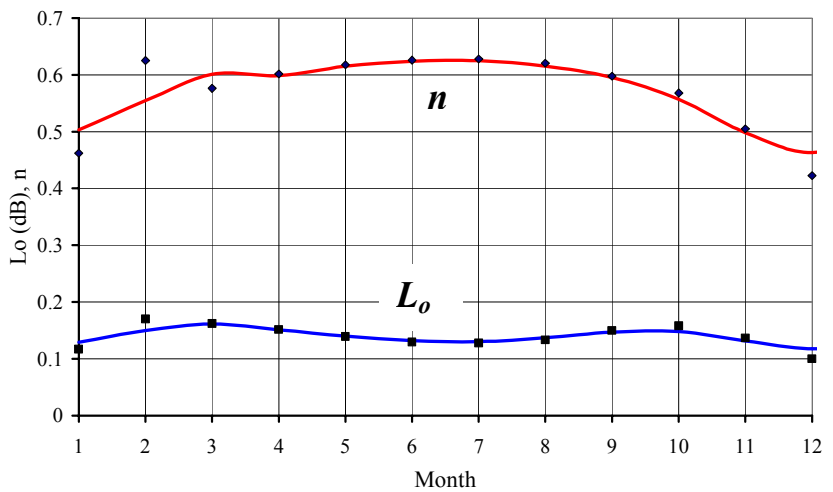

Fig. 5. Variation of the subsolar absorption Lo and the exponent $n$ as a function of season for low solar activity $\left(F_{10.7}=67 \mathrm{Jy}\right)$. The values are derived from the zenith angle ranges actually occurring in each month.

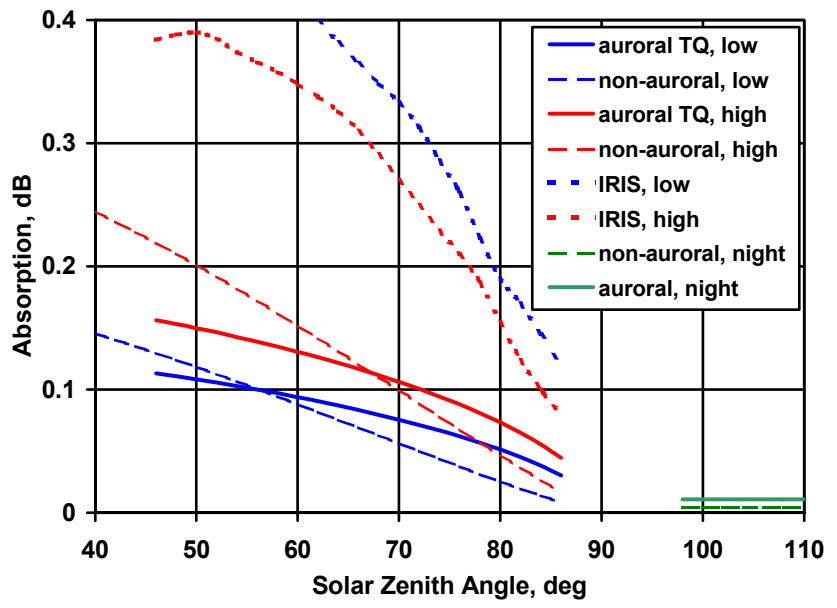

Fig. 6. Variation of the background absorption with solar zenith angle determined by different approaches (27.6 MHz, x-mode).

procedure for pressure surfaces in $20 \%$ increments starting from the top at $160 \mathrm{~km}$. Below about $90 \mathrm{~km}$, however, the electron densities under quiet conditions are below the EISCAT threshold and another source of electron densities has to be sought. From the Soviet Antarctic station Molodezhnaya 244 rocket borne probe measurements are available at three altitudes $(75,80$ and $84 \mathrm{~km})$. Because the neutral atmosphere in the Antarctic seems to be indistinguishable from that of the Arctic (Lbken et al., 1999), we feel comfortable to include these data from the "wrong" hemisphere in our analysis. Figure 4 shows True Quiet electron density profiles thus obtained for a medium solar activity $\left(F_{10.7}=130 \mathrm{Jy}\right)$ for various solar zenith angles. We now simulate integral absorption of a $27.6 \mathrm{MHz}$ signal (x-mode) using electron densities such as the ones shown in Fig. 4. The absorption $L$ obtained in this way is a function of solar zenith angle which again can be assimilated as $L=L_{o}(\cos \chi)^{n}$. Because in Winter months even the smallest $\chi$ are near or even beyond $90^{\circ}$, we replace $\cos \chi$ by $1 / \mathrm{Ch} \chi$. Figure 5 shows $L_{o}$ and $n$ calculated for each month using the zenith angle range actually occur-
Table 1. Representative annual mean parameters to estimate quiet absorption (27.6 MHz, x-mode, vertical incidence) for low and high solar activity, and auroral and non-auroral latitudes, respectively

\begin{tabular}{ccccc}
\hline & \multicolumn{2}{c}{ low solar activity (67 Jy) } & \multicolumn{2}{c}{ high solar activity (200 Jy) } \\
\hline & auroral & non-auroral & auroral & non-auroral \\
\hline$L_{o}, \mathrm{~dB}$ & 0.140 & 0.199 & 0.191 & 0.329 \\
$n$ & 0.570 & 1.175 & 0.546 & 1.114 \\
$L_{n}, \mathrm{~dB}$ & 0.012 & 0.004 & 0.012 & 0.004 \\
\hline
\end{tabular}

ring in that month (smoothed by a running mean). In Table 1 annual mean values of $L_{o}$ and $n$ are given for high and low activity, for other solar activities one can interpolate $L_{o}$ and $n$ for the desired solar fluxes. Also given in the table are the corresponding values from a non-auroral model of the lower ionosphere (Friedrich and Torkar, 2001) which are generally lower for those zenith angles where the auroral results are valid. The auroral zone night value $L_{n}$ is based on the lowest electron density ever measured by a sounding rocket at high latitudes (payload NLTE-2, ESRANGE, 6 March 1998, $\chi=117^{\circ}$ ). Figure 6 graphically shows the variations of the background absorptions with solar zenith angle.

\section{Conclusions}

Three methods to experimentally determine the absorption of the quiet ionosphere at high latitudes are presented, which are all based on reasonable arguments. However only the simulations using True Quiet electron density profiles yield results qualitatively in agreement with theoretical considerations. Electron densities for quiet night time conditions are far be- low the threshold of EISCAT, but the lowest rocket borne electron density profile can be used as a proxy for True Quiet beyond solar zenith angles of $98^{\circ}$.

\section{References}

Friedrich, M., Finsterbusch, R., Torkar, K. M., and Spöcker, P.: A Further Generalisation of the Sen and Wyller Magneto-Ionic Theory, Adv. Space. Res. 11 (10), 10 105-10 108, 1991.

Friedrich, M., Harrich, M., Torkar, K. M., and Stauning, P.: Quantitative Measurements with Wide-Beam Riometers, J. atmos. solar terr. Phys. 64, 359-365, 2002.

Friedrich, M. and Torkar, K. M.: FIRI: A Semiempirical Model of the Lower Ionosphere, J. geophys. Res. 106 (A10), 21409 $21418,2001$.

Lübken, F.-J., Jarvis, M. J., and Owen, G.: First in situ Temperature Measurements at the Antarctic Summer Mesopause, Geophys. Res. Lett. 26 (24), 3 581-3 584, 1999.

Phelps, A. V. and Pack, J. L.: Electron Collision Frequencies in Nitrogen and in the Lower Ionosphere, Phys. Rev. Lett. 3, 340$342,1959$.

Sen, H. K. and Wyller, A. A.: On the Generalization of the Appleton-Hartree Magnetoionic Formulas, J. geophys. Res. 65 (12), 3 931-3 950, 1960. 